\title{
The Influence of
}

\section{Acanthamoeba-Legionella Interaction in the Virulence of Two Different Legionella Species}

\section{Thiago Santos Gomes 1,2, Julia Gjiknuri', Angela Magnet', Lucianna Vaccaro', Dolores Ollero', Fernando Izquierdo', Soledad Fenoy', Carolina Hurtado ${ }^{1 \dagger}$ and Carmen del Águila ${ }^{*+}$}

${ }^{1}$ Facultad de Farmacia, Universidad San Pablo CEU, CEU Universities, Madrid, Spain, ${ }^{2}$ CAPES Foundation, Ministry of Education of Brazil, Brasilia, Brazil

The genus Legionella comprises more than 60 species, and about half are associated with infection. Legionella pneumophila is the most commonly associated with these infections and by far the most studied, but $L$. non-pneumophila species, such as L. feeleii, L. anisa, etc., may also present clinical importance. Free-living amoebae are their preferred environmental host, where these bacteria not only survive but also succeed in multiplying, and this relationship can lead to an increase in bacterial virulence. The goal of this study was to evaluate the alterations of Legionella pathogenicity due to its interaction with Acanthamoeba. For this, the expression of protein effectors SdhA, LegK2, and SidK were evaluated in L. pneumophila and L. feeleii, before and after infecting Acanthamoeba. Additionally, the host response was evaluated by measuring the production of IL-6, IL-8, and IFN- $\gamma$ in infected macrophages. Regarding the virulence factors, an increase in SdhA expression was observed after these bacteria infected Acanthamoeba, with a higher increase in the macrophage cultures infected with $L$. feeleii. Also, an increase in the expression of LegK2 was observed after infecting Acanthamoeba, but it was more intense in the cultures infected with $L$. pneumophila. With regard to SidK, it was increased in L. feeleii after infecting Acanthamoeba, however the same effect was not observed for $L$. pneumophila. In cytokine production, the effect on IL-6 and IL-8 was similar for both cytokines, increasing their concentration, but higher production was observed in the cultures infected with L. feeleii, even though it demonstrated slightly lower production with the inoculum obtained from Acanthamoeba. Concerning IFN- $\gamma$, induction was observed in both species but higher in the infection by L. pneumophila. Nevertheless, it is not known if this induction is enough to promote an efficient immune response against either $L$. pneumophila or $L$. feeleii. Altogether, these alterations seem to increase $L$. feeleii virulence after infecting Acanthamoeba. However, this increase does not seem to turn $L$. feeleii as virulent as L. pneumophila. More studies are necessary to understand the aspects influenced in these bacteria by their interaction with Acanthamoeba and, thus, identify targets to be used in future therapeutic approaches.

Keywords: Legionella, Acanthamoeba, interaction, virulence, Dot/lcm secretion system 


\section{INTRODUCTION}

Legionella spp. are Gram-negative bacteria with strict growth requirements. The genus comprises more than 60 species that have been isolated from aqueous environments. About half of the known species were also isolated at least once from patients, being thus associated with infection (Cunha et al., 2016; Khodr et al., 2016). Among these species, Legionella pneumophila is the most commonly associated with these infections and by far the most studied (Misch, 2016).

Free-living amoebae (FLA) are their preferred environmental host. A large variety of bacterial species have demonstrated their ability to survive and/or multiply within different amoebae, and thus may bypass disinfection treatments when harbored within amoebal cysts, leading to bacterial dispersion into the environment. Due to this feature, amoebic strains, mainly Acanthamoeba, have been used in laboratories as host cells to isolate amoeba-resisting bacteria (ARB) from complex samples (Laguna et al., 2006; Corsaro et al., 2009, 2010; Garcia et al., 2013). Moreover, pathogenic bacterial strains emerge from the environment after intracellular replication inside phagocytic protozoans, since bacterial replication within alveolar macrophages seems to mimic the relationship of these bacteria with amoebae. Hence, it has been proposed that its ability to employ basic cellular mechanisms in order to survive numerous protozoan hosts enables Legionella to infect human alveolar macrophages and cause disease (Laguna et al., 2006; Hervet et al., 2011; Michard et al., 2015; Sherwood and Roy, 2016). Therefore, the interaction between Legionella and FLA could play an important role in the ecology and pathogenicity of these bacteria. However, the number of bacteria isolated from water sources of transmission to humans during outbreaks of Legionnaire's disease is usually low or undetectable, so an enhanced infectivity of Legionella as a consequence of survival and replication within amoebae could compensate for the low infectious dose in the water sources (Richards et al., 2013).

After bacterial uptake, the endosomes containing Legionella are reported to follow a different route that bypasses the endocytic pathway and associates with endoplasmic reticulum-derived vesicles, forming the Legionella-containing vacuole (LCV), a rough endoplasmic reticulum-like compartment permissive for its intracellular replication. The morphology of the LCV in both mammalian cells and amoebae is similar, which supports the idea that there is an evolutionarily conserved pathway for promoting intracellular growth and suggests that amoeba serves as the "training site" for its ability to colonize higher organisms (Laguna et al., 2006; Zhu et al., 2011; Michard et al., 2015). The formation of the LCV is dependent on a type IVB secretion system (T4SS) known as the defective in organelle trafficking/intracellular multiplication (Dot/Icm) secretion system. This Dot/Icm T4SS translocates more than 300 bacterial proteins, called effectors, into the host cell and is absolutely required to reprogram the endosomal-lysosomal degradation pathway of the phagocytic cell and to trigger the biogenesis of the replication vacuole (Hervet et al., 2011; Harding et al., 2013; Michard et al., 2015).

On the other hand, there is an immunological consequence to this ability to subvert host cell trafficking via secreted effectors.
Apart from detecting the pathogen-associated molecular patterns (PAMPs), the innate immune system also detects perturbations of cellular physiology (Misch, 2016). The alveolar epithelial cells and macrophages play a critical role in preventing infection in mammalian lungs, sensing pathogen presence and inducing cytokine secretion to cause inflammation. However, proinflammatory cytokine production is a double-edged sword for the host, it works as a defense response when produced moderately but, when produced excessively, it may act as a pathogenic factor. After all, the pathogenesis of pneumonia is due to both host cell injury by bacterial virulence and its ability to induce host inflammation (Wang et al., 2015).

Previous environmental studies conducted on waters from drinking water treatment plants (DWTP) of Central Spain detected a high environmental presence of Acanthamoeba spp. in this region. Moreover, the presence of Legionella spp. was also reported in some of these samples. Although it did not detect the presence of L. pneumophila in these water samples, the presence of $L$. non-pneumophila species was reported both in raw and treated water. Most of these detections were succeeded by polymerase chain reaction (PCR), but an isolate of $L$. feeleii was able to be recovered by co-culture with Acanthamoeba (Magnet et al., 2012, 2015). Therefore, the successful isolation of viable L. feeleii bacteria led to a significant interest in understanding if the environmental presence of this species could represent a risk. After all, L. feeleii has already been reported as a causative agent of both Pontiac Fever and Legionnaire's disease (Muder and Yu, 2002; Siegel et al., 2010). This previous isolation of viable L. feeleii in water through amoebae co-culture and its reported capability of causing disease encouraged us to comparatively analyze some aspects of the virulence of L. pneumophila and L. feeleii, and also the possible alterations due to their interaction with Acanthamoeba.

\section{MATERIALS AND METHODS}

\section{Bacteria}

Two strains of Legionella were used in this study: (i) L. pneumophila strain JR32 and (ii) L. feeleii, environmental isolate from natural pool, obtained in a previous study (Magnet et al., 2015). They were cultured in AYE medium (Feeley et al., 1979), with some modifications. The composition is as follows: $10.0 \mathrm{~g}$ of yeast extract, $55 \mathrm{mM}$ ACES, $3.3 \mathrm{mM}$ L-cysteine, $0.6 \mathrm{mM}$ $\mathrm{Fe}\left(\mathrm{NO}_{3}\right)_{3} .9 \mathrm{H}_{2} \mathrm{O}$ and $5 \mu \mathrm{g} / \mathrm{mL}$ Chloramphenicol. Final $\mathrm{pH}$ was adjusted to 6.9 . Both strains were cultured in orbital agitation for $22 \mathrm{~h}$ at $37^{\circ} \mathrm{C}$, and dilutions were prepared when the culture reached $\mathrm{OD}_{600} \geq 3$. These conditions assured bacteria were at stationary growth phase, characterized for its higher infective capacity.

\section{Acanthamoeba spp.}

Acanthamoeba ATCC 30234, a genotype T4 strain free of Legionella was used in this study. This strain was cultured in axenic PYG medium (0.75\% proteose peptone, $0.75 \%$ yeast extract, and $1.5 \%$ glucose with $40 \mu \mathrm{g}$ gentamicin per milliliter) at $28^{\circ} \mathrm{C}$ without shaking. 


\section{Cell Culture}

RAW 264.7 (ATCC $^{\circledR}$ TIB- $71^{\mathrm{TM}}$ ), a cell line of murine macrophages, was used in this study and cultured in RPMI 1640 medium $\left(\mathrm{Gibco}^{\circledR}\right)$, supplemented with $10 \%$ fetal bovine serum (FBS), at $37^{\circ} \mathrm{C}$.

\section{Acanthamoeba Infection Assays}

The amoebae infection assays were performed as described by Declerck et al. (2007), with some modifications. These assays were carried out in 24-well tissue culture plates where $2 \times 10^{5}$ Acanthamoeba trophozoites were placed in $1.0 \mathrm{~mL}$ of Neff's saline per well. In these wells, a negative control without bacteria (Mock) and three different bacterial multiplicities of infection (MOI) were established, for each Legionella species: 1, 5, and 10. The plates were incubated at $33^{\circ} \mathrm{C}$ for $90 \mathrm{~min}$ and a subsequent 1 -h incubation with gentamicin $(100 \mu \mathrm{g} / \mathrm{mL})$ was conducted to eliminate remaining extracellular bacteria (i.e., bacteria that failed to infect a trophozoite before gentamicin incubation). Then, three washing steps were performed with $1 \mathrm{~mL}$ of Neff's saline per well. This time was set as time zero for post-infection bacterial recovery at $6,12,24$, and $48 \mathrm{~h}$ post-infection (hpi). These assays were in performed in triplicate.

\section{Preparation of Legionella Inocula Recovered From Acanthamoeba}

After evaluating the most efficient infection conditions observed at the Acanthamoeba infection assays, $10^{7}$ Acanthamoeba trophozoites were cultured in a $75 \mathrm{~cm}^{2}$ flask with Neff's saline and an infection procedure with the elected conditions (i.e., hpi and MOI) was conducted with L. pneumophila and L. feeleii, separately. At the end of the infection step, the flasks were incubated on ice to detach the amoebae from the culture well. Harvested cultures were concentrated by centrifugation $(1,000 \mathrm{rpm} / 10 \mathrm{~min})$ and resuspended in $100 \mu \mathrm{L}$, in which a volume of $300 \mu \mathrm{L}$ of $0.05 \%$ Triton X-100 (Sigma-Aldrich Chemie $\mathrm{GmbH}$, Germany) was added to promote amoebic lysis and intracellular bacteria release. From this solution, $100 \mu \mathrm{L}$ was inoculated in BCYE agar (with L-cysteine) to recover viable bacteria and incubated at $37^{\circ} \mathrm{C}$ for 3 days or until bacterial colonies were observed.

\section{Macrophage Infection Assays}

These assays were carried out in 24-well tissue culture plates where $2 \times 10^{5}$ cells were placed in $1.0 \mathrm{~mL}$ of RPMI 1640 supplemented with $10 \%$ FBS, per well. In these wells, a negative control without bacteria (Mock) and four different bacterial inocula were employed to establish infection: $L$. pneumophila from BCYE agar culture plates, L. pneumophila inocula recovered from Acanthamoeba, L. feeleii from BCYE agar culture plates and $L$. feeleii inocula recovered from Acanthamoeba. The macrophage infection was performed similarly as described for the Acanthamoeba infection assays, but only the most efficient conditions observed in these assays (MOI 1 at the infection step and $24 \mathrm{hpi}$ incubation afterward) were used. Once the infection step was established (bacterial incubation, gentamicin incubation, and washing steps), these plates were incubated at $37^{\circ} \mathrm{C}$ for $24 \mathrm{hpi}$. After this incubation, each culture was transferred to a $5.0 \mathrm{~mL}$ tube and concentrated by centrifugation at $1500 \mathrm{rpm} / 10 \mathrm{~min}$, separating supernatant and pellet for further analysis. These assays were in performed in triplicate.

\section{Determination of Pro-inflammatory Cytokines}

For this analysis, the culture supernatant of each well was submitted to an ELISA assay to determine the concentration of pro-inflammatory cytokines IL-6, IL-8, and IFN- $\gamma$. These assays were conducted using Mouse IL-6 (Interleukin 6) ELISA Kit - E-EL-M0044 (Elabscience Biotechnology, Co., Ltd.), Mouse IL-8 (Interleukin 8) ELISA Kit and Mouse IFN- $\gamma$ (Interferon Gamma) ELISA Kit - E-ELM0048 (Elabscience Biotechnology, Co., Ltd.), according to manufacturer's instructions. The cytokine amount was determined by comparing absorbance of each well to a standard curve and inferring values using the CurveExpert Basic 1.7 software.

\section{Determination of Legionella Protein Effectors Expression Elected Protein Effectors}

To assess the expression of bacterial virulent factors, three protein effectors translocated by the Dot/Icm T4SS were selected due to their reported role in Legionella's infection: SdhA, LegK2, and SidK. The justification for the specific selection of these genes was based on their reported role in this interaction, briefly described as follows. SdhA is one of the few Dot/Icm effectors that are crucial for the replication of L. pneumophila in mouse macrophages, as it is required to maintain the integrity of the LCV and thus avoid the activation of the host cell death (Creasey and Isberg, 2012; Harding et al., 2013). LegK2 is a protein factor that plays an essential role in bacterial survival by inhibiting actin polymerization on the LCV, evading endocytic degradation by preventing late endosome/lysosome association with the phagosome (Michard et al., 2015). SidK is

TABLE 1 | Selected protein effectors and the sequence of the specific primers designed.

\begin{tabular}{|c|c|c|c|}
\hline Protein effector & Primers & Primers sequences & Fragment size \\
\hline \multirow[t]{2}{*}{ SdhA } & SdhA $5^{\prime}$ & $\begin{array}{l}\text { 5'-ATG ATT TCA GAA AAG } \\
\text { ATC AAG CT-3' }\end{array}$ & $1,580 \mathrm{bp}$ \\
\hline & SdhA $3^{\prime}$ & $\begin{array}{l}5^{\prime}-T \text {-TA TGC GGA TGG } \\
\text { CGC TAA TTG GTT T-3' }\end{array}$ & \\
\hline \multirow[t]{2}{*}{ LegK2 } & LegK2 5' & $\begin{array}{l}\text { 5'-ATG GTT TAT TAC ATA } \\
\text { AAT TTG AAG-3' }\end{array}$ & $1,420 \mathrm{bp}$ \\
\hline & LegK2 3' & $\begin{array}{l}5^{\prime}-T \text { TA GCT TGG GCC } \\
\text { TCG CAT CAA-3' }\end{array}$ & \\
\hline \multirow[t]{2}{*}{ SidK } & SidK $5^{\prime}$ & $\begin{array}{l}5^{\prime}-T T G \text { TCT TाT ATC AAG } \\
\text { GTA GGT ATA-3' }\end{array}$ & $580 \mathrm{bp}$ \\
\hline & SidK $3^{\prime}$ & $\begin{array}{l}\text { 5'-AAA GGC TTA GGC TाT } \\
\text { CTा CC-3' }\end{array}$ & \\
\hline
\end{tabular}


a protein effector that inhibits vacuole acidification, interfering with the ability of the host cells to digest pathogens ( $\mathrm{Xu}$ et al., 2010).

\section{RNA Extraction}

The culture pellets derived from the macrophage infection assays were resuspended in $1.0 \mathrm{~mL}$ of Trizol ${ }^{\circledR}$ Reagent (Ambion, Life Technologies, Carlsbad, CA, United States), and RNA extraction was performed according to manufacturer's instructions. RNA concentration was measured using a GE NanoVue Spectrophotometer (GE Healthcare Life Sciences, United Kingdom).

\section{Reverse Transcription-Polymerase Chain Reaction}

A reverse transcription reaction was performed from $120 \mathrm{ng}$ of extracted RNA using the Thermo Scientific RevertAid H Minus First Strand cDNA Synthesis kit (Thermo Fisher Scientific, United States), according to manufacturer's instructions. Then, the obtained cDNA was used in a conventional PCR reaction designed to amplify specific fragments of the selected protein effector genes. The specific primers designed for these reactions are shown in Table 1. The PCR was performed in a total volume of $50 \mu \mathrm{L}$, using $1 \mathrm{X}$ Phusion Flash High-Fidelity PCR Master Mix (Thermo Fisher Scientific, United States), specific primers $0.2 \mu \mathrm{M}$ and $2 \mu \mathrm{L}$ of cDNA. The PCR reaction was carried out using a GeneAmp ${ }^{\circledR}$ PCR System 9700 thermocycler (Applied
Biosystems, United States), and the amplification parameters were as follows: $3 \mathrm{~min}$ at $95^{\circ} \mathrm{C} ; 40$ cycles of $1 \mathrm{~min}$ at $95^{\circ} \mathrm{C}$, $1 \mathrm{~min}$ at $55^{\circ} \mathrm{C}(\mathrm{SidK})$ or $60^{\circ} \mathrm{C}(\mathrm{SdhA}$ and $\operatorname{LegK} 2)$, and $2 \mathrm{~min}$ at $72^{\circ} \mathrm{C}$; with a final extension step of $3 \mathrm{~min}$ at $72^{\circ} \mathrm{C}$. The amplified product was visualized after electrophoresis in a $2.0 \%$ agarose gel. The visualized fragments were quantified by densitometry using Image J software (National Institutes of Health, United States). Additionally, it was performed RT-PCR assays designed to amplify a constitutive gen from mice [glyceraldehyde-3phosphate dehydrogenase (GADPH)] in order to assure these extracted RNA samples parted from approximately the same number of cells and thus standardize RNA concentration for these comparative analyses between in vitro models. Specific primers designed for this reaction were GADPH Forward (5'TGA GGC CGG TGC TGA GTA TGT CG-3') and GADPH Reverse (5'-CCA CAG TCT TCT GGG TGG CAG TG-3').

\section{RESULTS}

\section{Gene Expression of SdhA, LegK2, and SidK by Legionella, Before and After Infecting Acanthamoeba}

In Acanthamoeba infection assays, the expression of these protein effectors was evaluated to determine the most

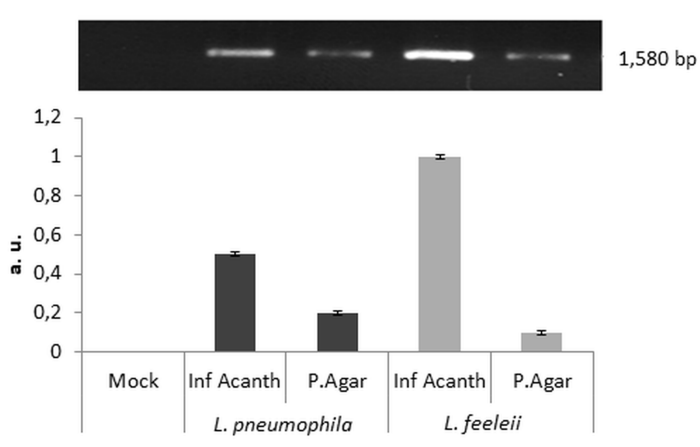

SdhA

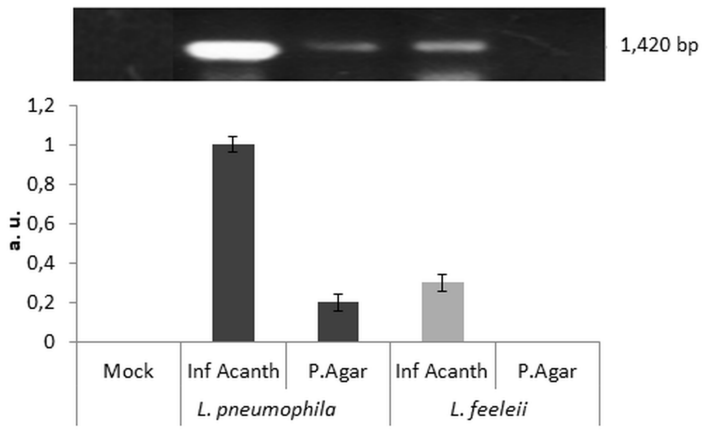

LegK2

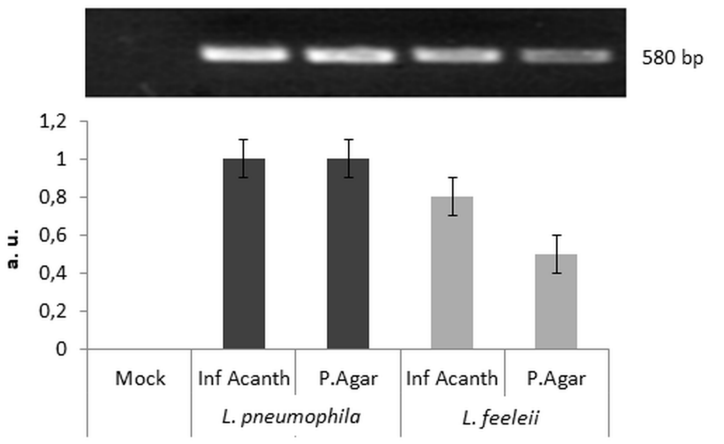

SidK

FIGURE 1 | Gene expression of protein effectors SdhA, LegK2, and SidK in macrophage infection, obtained through RT-PCR. The expression of these protein effectors was compared between L. pneumophila and L. feeleii, with an inoculum from agar culture and another recovered from Acanthamoeba infection (bps, base pairs; Inf Acanth, infection of Acanthamoeba; Mock, negative control; P. Agar, agar plate; a.u., arbitrary units). 

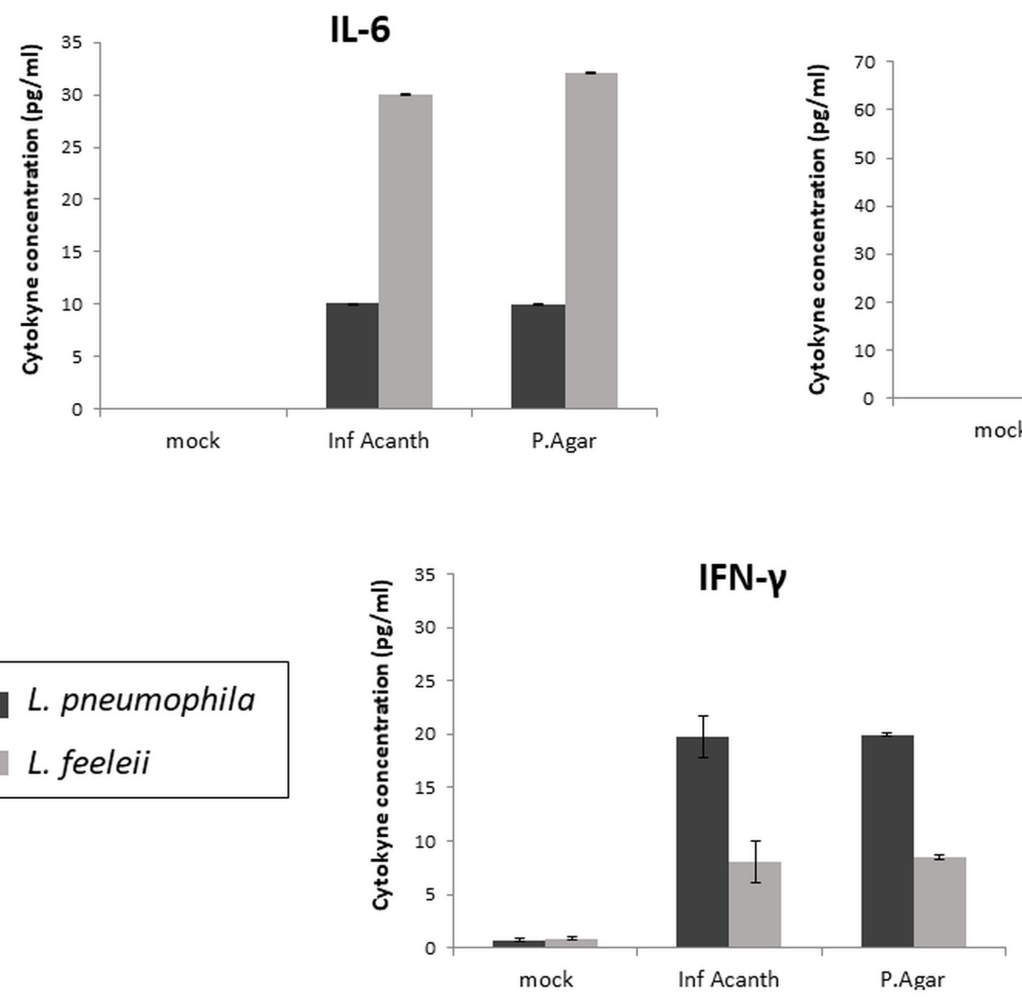

FIGURE 2 | Concentration of cytokines IL-6, IL-8, and IFN- $\gamma$ in macrophage infection, obtained through ELISA. The production of these cytokines was compared in infections from L. pneumophila and L. feeleii, with an inoculum from agar culture and another recovered from Acanthamoeba infection (Inf Acanth, infection of Acanthamoeba; Mock, negative control; P. Agar, agar plate).

efficient conditions in order to establish the previous Legionella-Acanthamoeba infection. For both species, the MOI 1 demonstrated to be the best option to recover bacterial inoculum and, at the time of infection of $24 \mathrm{hpi}$, gene expression demonstrated to occur more intensely (Supplementary Figure S1).

In the macrophage infection assays, RAW 264.7 cells were infected with both species and, for each of the species, an inoculum from agar and another recovered from Acanthamoeba. As additional information, a cytopathogenicity analysis and photographs of these macrophage infection assays can be seen in Supplementary Figure S2 and Supplementary Figure S3, respectively. Concerning the gene expression, the results obtained can be seen in Figure 1. In general, these data revealed that, except for SidK, the protein effectors demonstrated to be more intensely expressed at infection by bacterial inocula recovered from a previous infection in Acanthamoeba trophozoites. For protein effector SdhA, L. pneumophila from agar culture indicated a slightly higher expression than L. feeleii from agar. However L. feeleii recovered from Acanthamoeba demonstrated to not only increase its expression but also in a more intense way than L. pneumophila amoebic inoculum. For protein effector LegK2, a higher gene expression was observed in infections by $L$. pneumophila than L. feeleii, a result noticed for both agar or Acanthamoeba inoculum of each species. For SidK, L. pneumophila expression was not altered by the origin of the inoculum while $L$. feeleii expression demonstrated to be higher at the infection produced by the inoculum recovered from Acanthamoeba.

\section{Proinflammatory Cytokines Concentration From Macrophage Cultures Infected With Legionella, Before and After Infecting Acanthamoeba}

The aim of this analysis was to determine possible alterations in inflammatory response from macrophages when infected by Legionella from agar culture and by Legionella derived from a previous intracellular passage through Acanthamoeba. The results can be seen in Figure 2. Regarding IL-6 production, a higher concentration was observed in response to the presence of both species when compared to the uninfected control (Mock), but $L$. feeleii infection stimulated a more intense cytokine production than L. pneumophila. Nevertheless, the L. feeleii inoculum recovered from Acanthamoeba demonstrated to stimulate a slightly lower IL-6 production than bacteria from the agar culture, while L. pneumophila inocula recovered from Acanthamoeba stimulate similar levels of IL-6 production if compared to infection with bacteria from the agar culture. Regarding IL-8 production, the results were similar to those observed with IL-6. For IFN- $\gamma$ production, a higher concentration was observed in response to both species presence 
when compared to the negative control, but L. pneumophila infection seemed to stimulate a more intense cytokine production than L. feeleii. With regard to the inoculum, its origin did not demonstrate to influence IFN- $\gamma$ production.

\section{DISCUSSION}

The capability of intracellular bacteria such as Legionella spp. to survive and replicate depends on the adequate expression of genes that allow a higher success rate on the establishment of the infection of host cells and for this reason these genes are known as virulence genes. Regarding Legionella spp., an important fraction of its pathogenicity is attributed to the activity of effectors translocated by the Dot/Icm T4SS (Zhu and Luo, 2016). Taking into account the virulence produced by these pathogen effectors, three genes belonging to the Dot/Icm T4SS with a described role in Legionella intracellular survival were selected for this study.

SdhA is one of the few Dot/Icm effectors that seems to be crucial for the replication of $L$. pneumophila in mouse macrophages, being required for maintaining the integrity of the LCV. Disruption of the vacuole membrane during intracellular replication in macrophages is fatal to both the host cell and the bacterium. Destabilization of the LCV and release of the bacterium into the cytoplasm lead macrophages to rapid cell death due to the activation of pyroptosis, prematurely ending intracellular replication (Creasey and Isberg, 2012; Harding et al., 2013). Unlike apoptosis, which is often anti-inflammatory, pyroptosis is predicted to be proinflammatory, as it leads to a rapid loss of cell membrane integrity and release of cytosolic contents into the extracellular space, exposing pathogens to uptake and efficient killing by neutrophils through the activity of reactive oxygen species (Miao et al., 2010). In this study, SdhA expression was observed as more intense in L. pneumophila than L. feeleii in the inocula from agar culture, but the passage through Acanthamoeba produced a higher increase in the expression of this gene in L. feeleii than L. pneumophila. These data suggest that infecting Acanthamoeba activated the expression of this virulent protein effector in L. feeleii, more intensely.

LegK2 is a highly important Dot/Icm T4SS effector that controls actin polymerization remodeling on the LCV, contributing to Legionella evasion of endosome trafficking toward the LCV. This evasion of the endocytic pathway is the main virulence-related event of this bacterium infection cycle, preventing fusion with late endosomes and rerouting the phagosome to a replicative niche (Michard et al., 2015). In this study, LegK2 expression was observed as more intense in L. pneumophila than L. feeleii in both inocula, and the previous passage through Acanthamoeba produced an increase in the expression of this gene in these two Legionella species. These data could suggest that infecting Acanthamoeba increased the expression of this effector, very important in promoting the genesis of the LCV, in both species.

SidK is a protein effector that specifically targets host v-ATPase, the multi-subunit machinery responsible for organelle acidification in eukaryotic cells, and interacts with VatA, a key component of the proton pump. This interaction leads to the inhibition of ATP hydrolysis and proton translocation, decreasing LCV acidification and impairing the ability of host cells to digest its content. SidK expression seems to be highly induced when bacteria begin to enter a new growth cycle (Xu et al., 2010; Michard et al., 2015). In this study, SidK expression was observed as more intensely induced in L. pneumophila than L. feeleii in the inocula from agar culture. Curiously, the previous passage through Acanthamoeba produced an increase in the expression of this gene in L. feeleii, but no alterations were observed in the expression of this gene in L. pneumophila. Possibly, the ability of L. pneumophila to avoid LCV acidification was already an intrinsic characteristic acquired and highly expressed in the L. pneumophila strain used in this study, while the environmental $L$. feeleii strain did not express it so intensely and the interaction with Acanthamoeba provided an adverse condition in which a higher SidK expression was required for survival.

The higher expression of these protein effectors by inocula recovered from Acanthamoeba indicates that this Acanthamoeba-Legionella interaction might be crucial for increasing the pathogenesis of these bacteria, and these FLA may actually constitute a training site for both L. pneumophila and L. non-pneumophila species. Previous studies compared the invasive ability of bacteria grown in agar culture with that of bacteria grown in Acanthamoeba and observed that L. pneumophila recovered from Acanthamoeba were at least 100fold more invasive for epithelial cells and 10-fold more invasive for macrophages and amoebae than were L. pneumophila grown on agar. Then, it was suggested that these differences could be due to the expression of new proteins in amoebagrown L. pneumophila (Cirillo et al., 1994). This greater invasive ability acquired inside amoebae may be translated in a lower minimal infective dose and, thus, in a higher virulence. Regarding L. feeleii, there are no studies comparing virulence traits for this species between isolates from agar culture and from infected amoebae. However, it has already been shown that a L. feeleii isolate from a Legionnaire's disease patient presented a higher invasive capability than another isolate of the same strain of $L$. feeleii recovered from an individual suffering Pontiac fever (Wang et al., 2015). These differences of virulence traits on two isolates of the same strain of L. feeleii may be explained by an event that triggered an increase in virulence more intensely in one isolate than the another, and this event could be environmental interaction with free-living protozoa. In these interactions, the protein effectors assessed in the present study may play an important role in promoting a virulence increase, as well as in other effectors that remain to be studied.

Additionally, aspects of the virulence produced by host cells response were evaluated for the alterations produced as a consequence of pathogen activity. First, the proinflammatory cytokines interleukin-6 (IL-6) and interleukin-8 (IL-8) were selected due to their implication in the innate immune response. Cytokine IL-6 is a potent inducer of acute phase protein synthesis, whose levels in bronchoalveolar lavage (BAL) and serum are 
correlated, suggesting that IL-6 produced in the lung contributes at least in part to serum levels of this cytokine. This is an important feature since systemic levels of IL-6 can be correlated with the severity of the disease (Heinrich et al., 1990; Paats et al., 2013). Interleukin-8 exhibits a potent chemotactic activity for neutrophils and studies also suggest that by chemoattracting neutrophils it indirectly induces $\mathrm{CD} 4^{+} \mathrm{T}$ lymphocyte migration toward the site of infection. Like many inflammatory cytokine responses in community-acquired pneumonia, levels usually are higher in the lungs than in peripheral blood (Mukaida et al., 1998; Paats et al., 2013).

In this study, a similar effect was observed with regard to the production of IL- 6 and IL- 8 in the infected macrophages. Both cytokine concentrations were increased in the infected cells when compared to uninfected controls and their concentration in the cell cultures was higher in the cultures infected with L. feeleii, suggesting that this species might stimulate a more intense response. This could be due to a lower expression of the effectors involved in the escape of the local immune response. Regarding the different bacterial inocula, no alterations in IL-6 or IL-8 concentrations were observed for L. pneumophila infection, but a slight decrease in these cytokines concentration were observed in the cultures infected with $L$. feeleii recovered from Acanthamoeba when compared with bacterial inoculum from agar. These data suggest that L. pneumophila seems to succeed in partially inhibiting the proinflammatory response of the infected cells as described in the literature for the key immune proteins TNF, IL-6, IL-12, and CD86 (Copenhaver et al., 2015), and, for the strain evaluated in this study, this ability does not seem to be increased or decreased after interacting with Acanthamoeba. However, L. feeleii does not seem to exhibit such an ability in the present study, or at least not to the same degree. Although its passage through amoebae apparently causes a slight decrease on the immune response to its presence, the observed decrease does not reach the low levels produced in response to L. pneumophila infection.

The concentration of IFN- $\gamma$ was also evaluated for its important role in both innate and acquired immunity. An increase of IFN- $\gamma$ was observed in the presence of both species when compared to the negative control, but a higher increase was observed in the presence of L. pneumophila. Regarding the origin of the inocula, no alterations were observed for both L. pneumophila and L. feeleii. as a consequence of the previous passage through Acanthamoeba. Additionally, the observed increase in IFN- $\gamma$ concentration did not seem to be sufficient to stimulate a proper adaptive immune response, although it is not known if this effect is just a consequence of the lack of participation of other types of cells generating a more systemic type of response. Previous studies demonstrated that human macrophages were able to produce IFN- $\gamma$ when further stimulated with a combination of IL-12 and IL-18, or with macrophage colony-stimulating factor (M-CSF) (Darwich et al., 2009). In a cell culture containing macrophages exclusively, the co-stimulation generated by cytokines produced by other cells is absent. Previous studies have demonstrated that although macrophages are some of the prototypical producers of IL-12, they do not produce adequate quantities of IL-12 in response to Mycobacterium tuberculosis, for example. Furthermore, these studies demonstrated that cytokine interactions were important in restricting the growth of these Mycobacterium tuberculosis such us the reciprocal relationship of IFN- $\gamma$ on IL-18 signaling. IL-18 is known to influence IFN- $\gamma$ production in a number of cell types in both murine and human systems, but IFN$\gamma$ alone also seems to have an effect on IL-18 receptor gene expression, suggesting that other signals received by the macrophage during infection may be necessary to trigger a robust change in the IL-18 receptor at the cell surface (Robinson et al., 2012). Studies have emphasized the importance of the combined synergistic effect of IL-12 and IL-18, both in the differentiation of monocytes into mature macrophages, and in the subsequent stimulation of IFN- $\gamma$ production, and demonstrated that naturally activated alveolar macrophages immediately secreted IFN- $\gamma$ upon treatment with IL-12 and IL-18 (Darwich et al., 2009).

\section{CONCLUSION}

In summary, these data analyzed suggest that the previous passage through Acanthamoeba, and possibly other FLA, induce a higher expression of some virulence factors and, thus, the activation of mechanisms to provide the bacterium survival against immune responses. This feature seems to occur in both L. pneumophila and L. feeleii, but the alterations induced by Acanthamoeba appeared more intensely induced in L. feeleii than L. pneumophila. Thus, these alterations may convert L. feeleii to a more virulent bacterium after infecting Acanthamoeba. However, this species virulence increase does not seem to make L. feeleii as virulent as L. pneumophila. On the other hand, the higher immune response stimulated by $L$. feeleii infection may also lead to respiratory clinical symptoms and disease, since proinflammatory cytokine production works as a defense response when produced moderately but also acts as a pathogenic factor if exacerbated. It is important to understand the alterations produced on $L$. non-pneumophila species in their environmental interactions to increase awareness of the possibility of infection by those species, generally ignored in laboratorial diagnosis. This study is the first comparative analysis of $L$. feeleii virulence as a result of amoebic interaction and demonstrates the differences observed in this species in comparison to L. pneumophila, the most studied species. Nevertheless, more studies are still necessary to understand the entire set of factors influenced in these bacteria as a consequence of their interaction with Acanthamoeba and, thus, identify new targets and/or signaling pathways to be used in future therapeutic approaches.

\section{AUTHOR CONTRIBUTIONS}

TSG, CH, and CdÁ conceived and designed the experiments. TSG, JG, and $\mathrm{CH}$ performed the experiments. FI, SF, $\mathrm{CH}$, and CdÁ contributed with reagents, materials, and analysis tools. TSG wrote the first draft of the manuscript. $\mathrm{CH}$ and LV wrote sections 
of the manuscript and prepared figures. All authors analyzed the data, contributed to manuscript revision, and read and approved the submitted version.

\section{FUNDING}

This project was supported by Grants PI12/02725 and PI17/01670 from Instituto de Salud Carlos III (FISS) and FEDER and by grant USPCEU-PC07/2013 of the Fundación Universitaria San Pablo CEU. TSG was supported by CAPES/Brazil (Grant BEX 9132/13-9) and Lucianna Vaccaro by FPI USP-CEU.

\section{REFERENCES}

Cirillo, J. D., Falkow, S., and Tompkins, L. S. (1994). Growth of Legionella pneumophila in Acanthamoeba castellanii enhances invasion. Infect. Immun. 62, 3254-3261.

Copenhaver, A. M., Casson, C. N., Nguyen, H. T., Duda, M. M., and Shin, S. (2015). IL-1R signaling enables bystander cells to overcome bacterial blockade of host protein synthesis. Proc. Natl. Acad. Sci. U.S.A. 112, 7557-7562. doi: 10.1073/pnas.1501289112

Corsaro, D., Feroldi, V., Saucedo, G., Ribas, F., Loret, J. F., and Greub, G. (2009). Novel Chlamydiales strains isolated from a water treatment plant. Environ. Microbiol. 11, 188-200. doi: 10.1111/j.1462-2920.2008.01752.x

Corsaro, D., Pages, G. S., Catalan, V., Loret, J. F., and Greub, G. (2010). Biodiversity of amoebae and amoeba-associated bacteria in water treatment plants. Int. J. Hyg. Environ. Health. 213, 158-166. doi: 10.1016/j.ijheh.2010.03.002

Creasey, E. A., and Isberg, R. R. (2012). The protein SdhA maintains the integrity of the Legionella-containing vacuole. Proc. Natl. Acad. Sci.U.S.A. 109, 3481-3486. doi: 10.1073/pnas.1121286109

Cunha, B. A., Burillo, A., and Bouza, E. (2016). Legionnaires' disease. Lancet 387, 376-385. doi: 10.1016/S0140-6736(15)60078-2

Darwich, L., Coma, G., Peña, R., Bellido, R., Blanco, E. J., Este, J. A., et al. (2009). Secretion of interferon-gamma by human macrophages demonstrated at the single-cell level after costimulation with interleukin (IL)-12 plus IL-18. Immunology 126, 386-393. doi: 10.1111/j.1365-2567.2008.02905.x

Declerck, P., Behets, J., De Keersmaecker, B., and Ollevier, F. (2007). Receptormediated uptake of Legionella pneumophila by Acanthamoeba castellanii and Naegleria lovaniensis. J. Appl. Microbiol. 103, 2697-2703. doi: 10.1111/j.13652672.2007.03530.x

Feeley, J. C., Gibson, R. J., Gorman, G. W., Langford, N. C., Rasheed, J. K., Mackel, D. C., et al. (1979). Charcoal-yeast extract agar: primary isolation medium for Legionella pneumophila. J. Clin. Microbiol. 10, 437-441.

Garcia, A., Goñi, P., Cieloszyk, J., Fernandez, M. T., Calvo-Beguería, L., Rubio, E., et al. (2013). Identification of free-living amoebae and amoeba-associated bacteria from reservoirs and water treatment plants by molecular techniques. Environ. Sci. Technol. 47, 3132-3140. doi: 10.1021/es400160k

Harding, C. R., Stoneham, C. A., Schuelein, R., Newton, H., Oates, C. V., Hartland, E. L., et al. (2013). The Dot/Icm effector SdhA is necessary for virulence of Legionella pneumophila in Galleria mellonella and A/J mice. Infect Immun. 81, 2598-2605. doi: 10.1128/IAI.00296-13

Heinrich, P. C., Castell, J. V., and Andus, T. (1990). Interleukin-6 and the acute phase response. Biochem. J. 265, 621-636. doi: 10.1042/bj2650621

Hervet, E., Charpentier, X., Vianney, A., Lazzaroni, J. C., Gilbert, C., Atlan, D., et al. (2011). Protein kinase LegK2 is a type IV secretion system effector involved in endoplasmic reticulum recruitment and intracellular replication of Legionella pneumophila. Infect. Immun. 79, 1936-1950. doi: 10.1128/IAI.00805-10

Khodr, A., Kay, E., Gomez-Valero, L., Ginevra, C., Doublet, P., Buchrieser, C., et al. (2016). Molecular epidemiology, phylogeny and evolution of Legionella. Infect. Genet. Evol. 43, 108-122. doi: 10.1016/j.meegid.2016.04.033

Laguna, R. K., Creasey, E. A., Li, Z., Valtz, N., and Isberg, R. R. (2006). A Legionella pneumophila-translocated substrate that is required for growth

\section{ACKNOWLEDGMENTS}

The authors are indebted to Sergio Llorens for his valuable technical assistance and to Brian Crilly for his helpful revision of the manuscript.

\section{SUPPLEMENTARY MATERIAL}

The Supplementary Material for this article can be found online at: https://www.frontiersin.org/articles/10.3389/fmicb. 2018.02962/full\#supplementary-material

within macrophages and protection from host cell death. Proc. Natl. Acad. Sci. U.S.A. 103, 18745-18750. doi: 10.1073/pnas.0609012103

Magnet, A., Galván, A. L., Fenoy, S., Izquierdo, F., Rueda, C., Fernandez Vadillo, C., et al. (2012). Molecular characterization of Acanthamoeba isolated in water treatment plants and comparison with clinical isolates. Parasitol. Res. 111, 383-392. doi: 10.1007/s00436-012-2849-2

Magnet, A., Peralta, R. H., Gomes, T. S., Izquierdo, F., Fernandez-Vadillo, C., Galvan, A. L., et al. (2015). Vectorial role of Acanthamoeba in Legionella propagation in water for human use. Sci. Total Environ. 505, 889-895. doi: 10.1016/j.scitotenv.2014.10.064

Miao, E. A., Leaf, I. A., Treuting, P. M., Mao, D. P., Dors, M., Sarkar, A., et al. (2010). Caspase-1-induced pyroptosis is an innate immune effector mechanism against intracellular bacteria. Nat. Immunol. 11, 1136-1142. doi: 10.1038/ni. 1960

Michard, C., Sperandio, D., Baïlo, N., Pizarro-Cerdá, J., LeClaire, L., ChadeauArgaud, E., et al. (2015). The legionella kinase LegK2 targets the ARP2/3 complex to inhibit actin nucleation on phagosomes and allow bacterial evasion of the late endocytic pathway. mBio 6:e00354-15. doi: 10.1128/mBio. 00354-15

Misch, E. A. (2016). Legionella: virulence factors and host response. Curr. Opin. Infect. Dis. 29, 280-286. doi: 10.1097/QCO.0000000000000268

Muder, R. R., and Yu, V. L. (2002). Infection due to Legionella species other than L. pneumophila. Clin. Infect. Dis. 35, 990-998. doi: 10.1086/342884

Mukaida, N., Harada, A., and Matsushima, K. (1998). Interleukin-8 (IL-8) and monocyte chemotactic and activating factor (MCAF/MCP-1), chemokines essentially involved in inflammatory and immune reactions. Cytokine Growth Factor Rev. 9, 9-23. doi: 10.1016/S1359-6101(97)00022-1

Paats, M. S., Bergen, I. M., Hanselaar, W. E., Groeninx van Zoelen, E. C., Hoogsteden, H. C., Hendriks, R. W., et al. (2013). Local and systemic cytokine profiles in nonsevere and severe community-acquired pneumonia. Eur. Respir. J. 41, 1378-1385. doi: 10.1183/09031936.00060112

Richards, A. M., Von Dwingelo, J. E., Price, C. T., and Abu Kwaik, Y. (2013). Cellular microbiology and molecular ecology of Legionella-amoeba interaction. Virulence 4, 307-314. doi: 10.4161/viru.24290

Robinson, C. M., Jung, J. Y., and Nau, G. J. (2012). Interferon- $\gamma$, tumor necrosis factor, and interleukin-18 cooperate to control growth of Mycobacterium tuberculosis in human macrophages. Cytokine 60, 233-241. doi: 10.1016/j.cyto. 2012.06.012

Sherwood, R. K., and Roy, C. R. (2016). Autophagy evasion and endoplasmic reticulum subversion: the yin and yang of legionella intracellular infection. Annu. Rev. Microbiol. 70, 413-433. doi: 10.1146/annurev-micro-102215095557

Siegel, M. O., Fedorko, D. P., Drake, S. K., Calhoun, L. B., and Holland, S. M. (2010). Legionella feeleii serotype 2 pneumonia in a man with chronic lymphocytic leukemia: a challenging diagnosis. J. Clin. Microbiol. 48, 22942297. doi: 10.1128/JCM.00176-10

Wang, C., Saito, M., Tanaka, T., Amako, K., and Yoshida, S. (2015). Comparative analysis of virulence traits between a Legionella feeleii strain implicated in Pontiac fever and a strain that caused Legionnaires' disease. Microb. Pathog. 89, 79-86. doi: 10.1016/j.micpath.2015.09.004 
Xu, L., Shen, X., Bryan, A., Banga, S., Swanson, M. S., and Luo, Z. Q. (2010). Inhibition of host vacuolar $\mathrm{H}^{+}$-ATPase activity by a Legionella pneumophila effector. PLoS Pathog. 6:e1000822. doi: 10.1371/journal.ppat. 1000822

Zhu, W., Banga, S., Tan, Y., Zheng, C., Stephenson, R., Gately, J., et al. (2011). Comprehensive identification of protein substrates of the Dot/Icm type IV transporter of Legionella pneumophila. PLoS One 6:e17638. doi: 10.1371/ journal.pone. 0017638

Zhu, W., and Luo, Z. Q. (2016). Cell biology and immunology lessons taught by Legionella pneumophila. Sci. China Life Sci. 59, 3-10. doi: 10.1007/s11427-015$4945-\mathrm{x}$
Conflict of Interest Statement: The authors declare that the research was conducted in the absence of any commercial or financial relationships that could be construed as a potential conflict of interest.

Copyright (๔ 2018 Gomes, Gjiknuri, Magnet, Vaccaro, Ollero, Izquierdo, Fenoy, Hurtado and del Águila. This is an open-access article distributed under the terms of the Creative Commons Attribution License (CC BY). The use, distribution or reproduction in other forums is permitted, provided the original author(s) and the copyright owner(s) are credited and that the original publication in this journal is cited, in accordance with accepted academic practice. No use, distribution or reproduction is permitted which does not comply with these terms. 